\title{
A Comparative Review on Routing Protocols in Wireless Sensor Networks
}

\author{
Tarun Sharma \\ Department of Computer \\ science \& Engineering, CT \\ Instittute of Technology \& \\ Research, jalandhar, India
}

\author{
Harsukhpreet Singh \\ Department of Electronics \& \\ Communication, CT Institute of \\ Technology \& Research, \\ Jalandhar, India
}

\author{
Anurag Sharma \\ Department of Electronics \& \\ Communication, CT Institute of \\ Technology \& Research, \\ Jalandhar, India
}

\begin{abstract}
Wireless Networking is a technology in which number of mobile nodes can communicate with each other directly or indirectly through wireless links. A sensor network is composed of a large number of sensor nodes and a sink. In the wireless sensor networks the main problem is limited battery life used by sensor nodes because the size of sensor nodes is small so constraints are there like battery size, processor, storage for data, these all are small as sensor nodes Routing protocols of sensor networks are dependable for handles the routes in the networks. This paper surveys current routing protocols for sensor networks and present a categorization for the various approaches pursued and contrast their strength and constraint.
\end{abstract}

\section{General Terms}

Routing protocols, comparison of Routing protocols

\section{Keywords}

WSN (wireless sensor network), Routing protocol, classification of protocol.

\section{INTRODUCTION}

A wireless sensor network is made up of large number of sensor nodes and a sink [2]. The WSN is composed of sensor nodes from hundreds or thousands and each node is connected to one sensor nodes. A wireless sensor network made up of a large number of nodes extends over a specific area. A sensor node self-possessed of sensor, actuators, memory, a mainframe and they do have communication capacity. All the sensor nodes are permit to communicate in the course of a wireless intermediate. The wireless medium is of infrared, radio frequency that having no wired connection attached. So the sensor nodes are deployed in a random manner and it make ad-hoc network because they can communicate themselves [1]. If node is unable to communicate with other nodes of the network through direct link, it means node is out of range. In such kind of networks data transmission from one node to another is performed via in the middle of nodes. This concept is referred as multi-hoping. All sensors nodes work cooperatively to serve the requests [4].

The main problem in WSN, sensor node have limited battery life because the sensor nodes size is small so battery size, processor, storage space for data, these all are little as sensor nodes. In WSN a bundle of sensed information and routing information has to be sending which after have some time constraints so that information can be employable before any disaster occurs like manufacturing monitoring, apparatus monitoring etc. In WSN the power influence utilization is much superior data communication then internal processing. Energy preservation in WSN is need to the address [2]. Wireless sensor is flat to node breakdown due to power hammering. In order to provide a consistent service through network, the network should be personality adjusting and must have flexible properties as requisite from time to time. A restricted access node may encounter failure due to imperfect battery existence. In such case the network protocol should be intellectual enough to such collapse to keep the network operational many techniques are proposed for energy discount, clustering is single of them. The cluster heads are voted sporadically such that members of a cluster can communicate with their cluster heads. These cluster heads send data acknowledged from its members to a base station. The multi clustering can also be used. The cluster head should have to be rotated for the complementary of energy and then there will be equivalent weight on every node. The energy expenditure can be condensed [2].

In this paper discuss some mixture of routing protocols for wireless sensor networks. In section 2, the network routing issues and design challenges are described. In section 3 present related work. In section 4, compare and discussed routing protocols of WSN, finally and section 5 concludes the survey.

\section{NETWORK DESIGN CHALLENGES AND ROUTING ISSUES}

The design of routing protocol for WSNs is tricky because of numerous network limitations. WSNs experience from the restrictions of several network resources, for examples, energy, bandwidth, processor, and storage. The aim of challenges in sensor networks involves the following main aspects $[5,20]$.

\subsection{Restricted energy capacity}

The goal of routing protocols is to transfer data among sensors and sink. Sensor node consumes large amount of energy for sensing, processing, receiving and transmitting information. So data transmission is energy consuming. Since, the sensor node has limited energy because the size of sensor nodes is small so the data of storage is also small. So, there is a need to design routing protocols.

\subsection{Sensor locations}

Another limitation that faces the plan of routing protocols is to deal with the location of the sensor. Most of the designed protocols suppose that the sensor either are operational with global positioning system (GPS) receivers or use numerous location methods to learn about their location.

\subsection{Limited hardware resources}

In WSN has limited hardware resources, sensor node have limited energy capacity because the size of sensor node is small; processing and storage capacities can only perform limited function. So hardware constraints present much limitation in software advance and network protocol design for sensor nodes.

\subsection{Coverage}

A sensor's view of the environment is inadequate both in range and in correctness. Hence coverage area is essential design issue. 


\subsection{Data Aggregation}

Since sensor nodes may generate significance unneeded data, related packet from numerous nodes can be aggregated so that the quantity of broadcast is condensed. This technique has been used to attain energy effectiveness and data convey optimization in a number of routing protocol.

\subsection{Diverse Sensing application requirements}

Sensor networks have a wide-ranging variety of miscellaneous application. No network protocol can convene the supplies of all application therefore, routing protocols should agreement data release and its exactness so that the sink can meet the required awareness about the physical occurrence on time.

\subsection{Scalability:}

Routing protocols should be capable to extent with the network dimension. Also, sensor may not have the similar capabilities in conditions of energy, processing, sensing and communication. Hence, communication connection between sensors may not be symmetric, that is, a couple of sensor may not be competent to have communication in both directions. This should be taken concern of in routing protocols.

\section{LITRATURE REVIEW}

From the last few decades, different techniques and protocols have been proposed to improvement of WSN.

Sensor networks with feasible meeting of micro electromechanical systems tools, wireless communications and digital electronics concept described by Akyildiz I. et al.; [2002]. First, the sensing tasks and the probable sensor networks applications were explored, and reviewed factors influencing the design of sensor networks is provided. The flexibility, fault tolerance, high sensing fidelity, low-cost and rapid deployment characteristics of sensor networks create many new and exciting application areas for remote sensing[2].

Clustering routing algorithm which precedence to energy competence proposed by Jian-qi L. et al. (2013). First, spawn cluster head by casual competition in the nodes which have benefit in energy; next resolve the internal construction of clusters by manipulative dynamically rigidity coefficient of each cluster, after that, optimize broadcast path between cluster heads through improved multi-objective unit swarm algorithm [4].

An optimal energy-saving spare management, including spare selection and named it LEACH-SM protocol (modified form of prominent LEACH protocol) was proposed by Baker B. et al.; (2014).In this author presented a quantitative comparison of energy consumption and WSN lifetime for both mentioned protocols [6].

The FAF-EBRM protocol compared with LEACH protocol and this method is used for the next hop node selected according to the forward energy density and link weight EEUC proposed by Zhang et al.;(2014). The proposed method balance the energy reduction, function lifetime and provide good quality of service [3].

Hybrid clustering approach a cluster head reduce of its energy and clustering is used to beginning of the upcoming round proposed by Neamatollahi.P et al. (2010). Clustering is performed on demand. To elaborate the efficiency of proposal, the distributed clustering protocol HEED (Hybrid Energy Efficient Distributed) hybrid clustering algorithm is used as baseline example. Through simulation results, it shows that HCA is approximately $30 \%$ more efficient in terms of network lifetime than the other protocol. The main reason is that the clustering is executed on demand [8].
Genetic algorithm and optimization of LEACH protocol that are used on LEACH protocol and contrast both results on the basis of rounds that was discussed by Yadav S. et al.; (2014) . This comparison was based on optimal thresholding probability for cluster formation .Finally after comparison find LEACH-GA technique outperforms MTE,DT and LEACH in terms of network lifetime, use for optimal energy-efficient clustering [12] .

Threshold sensitive Energy Efficient sensor Network Protocol (TEEN) protocol was estimated by Manjeshwar A. et al.; (2001). Closer nodes form clusters, with a cluster heads to broadcast the collected information to one senior layer. Forming the clusters, cluster heads transmit threshold values. First one is hard threshold; it is least possible value of an attribute to trigger a sensor node. Hard threshold permits nodes convey the event, if the event happens within the range of interest. Thus a significant reduction of the transmission delay happens. Unless an amendment of least soft threshold happens, the node doesn't send a new data packet. Using soft threshold prevents from the redundant information/data transmission. Since the protocol is to be attentive to the quick changes in the perceived attribute; therefore, it is appropriate for time-critical applications [26].

The two important clustering protocols, namely LEACH and LEACH-C (centralized), using NS2 tool for frequent chosen scenarios, and study of simulation results against chosen performance metrics with latency and network lifetime was designed by Nayak P.et al.; (2014). As a termination of observation from results, it can be mentioned that LEACH can be preferred if localized coordination of nodes in clustering without involving BS is of high precedence than other factors like assurance over desired number of clusters etc.; and LEACH-C can be chosen when centralized and deterministic approach covering entire network is expected still bringing in increased network lifetime and desired number of clusters [7].

The modified version of LEACH protocol called V-LEACH protocol and the comparison of LEACH protocol with $\mathrm{V}$ LEACH protocol was planned by Alhawat A. et al.;([2013) From the simulation results were, first the number of alive nodes is more than the original LEACH. Second the number of dead nodes is less than the original LEACH protocol. Network life time is increased by $49.37 \%$ then original LEACH [27].

\section{ROUTING PROTOCOLS IN WSN}

Many routing algorithms were developed for wireless networks. All foremost routing protocols planned for WSNs may be divided into five categories as shown in table 1.we evaluation the model of routing protocols in each of the categories in previous sub-sections [21].

\subsection{Hierarchical protocols}

In this segment, we evaluation a sample of hierarchical-based routing protocols for WSNs. Hierarchical protocols is a cluster based protocols. Clustering is an energy-efficient communication protocols that can be used by the sensors to account their sensed data to the base station [9].

\subsubsection{Low-energy adaptive clustering hierarchy} (LEACH): Low Energy adaptive clustering hierarchical protocols. It is a routing protocols and also known as cluster based protocols. LEACH protocol provides communication between two sensor nodes in WSN. LEACH is most commonly used protocol in WSN [6]. 


$$
\begin{aligned}
& \mathrm{T}(\mathrm{n})=\frac{p}{1-p *\left(\operatorname{rmod} \frac{1}{p}\right)}: \text { if n E G } \\
& \mathrm{T}(\mathrm{n})=0 \quad: \text { Otherwise }
\end{aligned}
$$

Limitation in LEACH protocol is chosen of $\mathrm{CH}$ randomly is main problem of LEACH protocol because when $\mathrm{CH}$ is selected in randomly way then there is no record account for energy consumption. So a node with low energy has same probability as node of high energy. If node with low Energy is chosen as $\mathrm{CH}$ then this node will die soon due to which WSN cannot exist for a long time. There are some modified versions of LEACH protocol like V-LEACH protocol, M-LEACH protocol, ELEACH protocol, and O-LEACH protocol [23, 24, 25 ].

Table 1: Routing Algorithm in WSN

\begin{tabular}{|c|l|}
\hline Category & \multicolumn{1}{|c|}{ Representative protocols } \\
\hline $\begin{array}{c}\text { Hierarchical } \\
\text { protocols }\end{array}$ & $\begin{array}{l}\text { LEACH,PEGASIS,Layered } \\
\text { PEGASIS,HEED, } \\
\text { TEEN, APTEEN, TTDD. }\end{array}$ \\
\hline $\begin{array}{c}\text { Heterogeneity } \\
\text { based } \\
\text { protocols }\end{array}$ & IDSQ,CADR,CHR \\
\hline $\begin{array}{c}\text { Location - } \\
\text { based } \\
\text { protocols }\end{array}$ & $\begin{array}{l}\text { GEAR,GAF,Span,TBF,BVGF,GeRaF, } \\
\text { MECN,SMECN }\end{array}$ \\
\hline $\begin{array}{c}\text { Data-Centric } \\
\text { Protocols }\end{array}$ & $\begin{array}{l}\text { SPIN, Directed Diffusion (DD),Rumor } \\
\text { routing,COUGAR, ACQUIR, EAD }\end{array}$ \\
\hline $\begin{array}{c}\text { QoS-based } \\
\text { Protocols }\end{array}$ & $\begin{array}{l}\text { SAR,SPEED,MMSPEED,Energy-aware } \\
\text { routing }\end{array}$ \\
\hline
\end{tabular}

\subsubsection{Power-Efficient Gathering in sensor}

Information system (PEGASIS)

PEGASIS is an expansion of the LEACH protocol that contains shackles from sensor nodes so that each node send out and receives from a neighbor and only one node is nominated from that chain to transmit to the BS (Sink).The data is gathered and shift from node to node and sent to the BS. The series is performed in a greedy way. Unlike LEACH protocol, PEGASIS protocol avoids cluster arrangement and use only one node in a chain to send out to the BS (sink) instead of using compound nodes. In every round, preferred sensor node from the chain arbitrarily that will send out the aggregated data to the BS, then sinking the per round energy overheads compared to LEACH. Simulation outputs showed that PEGASIS is able to boost the life time of the network double as a lot the life span of the network under the LEACH protocol [11].

\subsubsection{Layered PEGASIS}

Layered PEGASIS protocol

Is an expansion of PEGASIS. Layered PEGASIS is mostly solving the difficulty of wait in data transmission caused by lengthy chain.

\subsubsection{HEED (Hybrid Energy-efficient Distributed Clustering)}

HEED extends the basic idea of LEACH by using residual energy as primary parameters and network topology features are only used as secondary parameters to crack tie between applicant cluster heads a metric for cluster collection to attain power balancing. HEED proposed was minimizing control overhead, producing well-distributed $\mathrm{CHs}$ and compact clusters .The HEED improves network lifetime over LEACH because $\mathrm{LEACH}$ arbitrarily elected $\mathrm{CHs}$, which may result in quicker death of some sensor nodes. The final Cluster head elected in HEED that is well spread across the network and the communication expenditure is minimized [8].

\subsubsection{Threshold sensitive energy efficient sensor network protocol (TEEN)}

TEEN is a cluster based algorithm. It is similar to LEACH protocol, in which most nodes transmit to $\mathrm{CHs}$, and the cluster head aggregate and compress the data and forward it to the base station (sink). TEEN is a routing protocols designed for reactive WSNs. significant features of TEEN include its correctness for time critical sensing application. Since the transmission of message consumes high energy than data sensing [5].

\subsubsection{APTEEN}

Is an development of TEEN protocol, which fiddle with the parameters issued by the cluster head, which can modify allied parameters according to the needs of users or the use of type, including a set of physical attributes expressed that users expect to get; hard and soft threshold; operation mode (TDMA); counting time (CT), the mainly time period represented unbeaten data transmission of a node [13].

\subsubsection{Two Tier Data Dissemination (TTDD protocol)}

TTDD is valid to multi-sink node and the sink node moving in the network. When multiple nodes sense events, a node is chosen as the source node to send data. The source node set itself as a cross-point of grid to build a Grid network, the process is: the source node determines the location of nearby cross-point first, requests the node closest becoming a new cross-point through using the greedy algorithm, the new intersection continues the process until the request expired or reaching the network border. Intersection saves the event and information of the source node. Data query, the sink node use flooding query technique to request the adjacent cross-node, then the query request transmitted in the cross-point, and eventually the source node receives a query request, the data will be send back to the sink node [18].

\subsection{Data-Centric protocols:}

Data-centric protocol is used to control the redundancy it happens because sensor node does not have global identification number which contains uniquely, so data is transmitted to each node with significant redundancy of data .In data centric routing, the sink request for data by sending the query so the nearest sensor node transmits the data selected understands from the query [14].

\subsubsection{Sensor protocols for Information via} Negotiation (SPIN)

SPIN protocol was proposed to beat the difficulty of flooding. In flooding sensor node transmits the data to its neighbors until data does not reach the destination. Some limitation of flooding is Implosion and overlap [15].

- Implosion: It is caused by duplicated message sent to the same neighbor's node.

- Overlap problem: The data is sent at the same time so the overlap problem is arises. so result is neighbors receiving duplicate reports of the same event

4.2.2Directed Diffusion: Directed diffusion is a datacentric and application awake model in the sense that all data 
generated by sensor nodes is named by attribute- importance pairs. The goal of Directed diffusion combines the data coming from different sources by eliminating redundancy, minimizing the number of transmission for saving network energy and prolonging its lifetime [14]

4.2.3Rumor Routing: Rumor routing performs fine only when the number of tasks is small and number of queries is small. Rumor routing use long lived packet known as agent, created at random nodes. it is also known as agent based algorithm .This routing is between query flooding and event flooding. It provides energy saving over flooding and easily handles node failure. But main problem of this routing is performs only when number of events is small [14].

Some protocols like ACQUIRE, EAD, Cougar these are also data centric protocols.

\subsection{Heterogeneity-based Protocols:}

Heterogeneity sensor networks are of two types of sensors namely line-powered and battery powered. In this segment we talk about uses of heterogeneity based protocols in WSNs to expand the network lifetime [22].

\subsubsection{Information-Driven Sensor Query (IDSQ):} IDSQ is used to detect the problem of heterogeneous WSN for gaining information, minimizing to detect latency and energy consumption. In IDSQ protocol, first is to select a sensor as leader from the cluster of sensors. This leader will be responsible for selecting optimal sensors based on some information utility measure [4].

\subsubsection{Cluster-Head Relay Routing(CHR): $\quad$ CHR} routing protocol is separated into two types of sensors namely large number of low-end sensors that is denoted by L-sensors and second small number of powerful high end sensors that is denoted by $\mathrm{H}$ - sensors. Both these sensors are static and aware of their locations using some location service. L-sensors use short-range data transmission range and $\mathrm{H}$-sensors perform long range data transmission [4].

4.4 Location-Based protocols: Sensors nodes are pointed by mean their positions. Position information for sensor nodes is required for the sensor networks by the majority of the routing protocols to add the distance between two exacting nodes so that energy expenditure can be reduced [16].

4.4.1Geographic Adaptive Fidelity (GAF): GAF is an Energy-aware routing protocol that is designed for MANETs, but can also be second-hand for WSNs in sense of energy management. GAF is highly scalable with limited power usage [17].

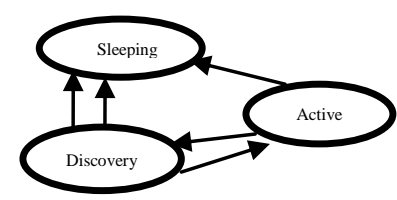

Fig. 3 State diagram of GAF

In this Figure 3, the state diagram of GAF has three levels, namely discovery, active and sleeping. In sleeping condition for energy savings its turns of its radio. In the discovery state, a sensor interactions discovery messages to study about further sensors in the identical grid. In the Active state, a sensor transmission its discovery message to inform matching sensors about its state.

\subsubsection{Geographic and Energy-Aware Routing} $(G E A R)$ : GEAR is a location based routing protocol, it has low scalability and provides demand driven data delivery. Gear proposed for routing queries to target region in a sensor like GPS component or a localization system so that they recognize their present position [17].

\subsubsection{Greedy Approach:}

In [10] I. Stojmenovicet.al stated that the neighboring node $\mathrm{X}$ which is closer to the destination node D from source or intermediate node $\mathrm{Y}$ conducts the packet to the destination. The data flows through the intermediate nodes until it will reach the destination node $\mathrm{D}[16]$.

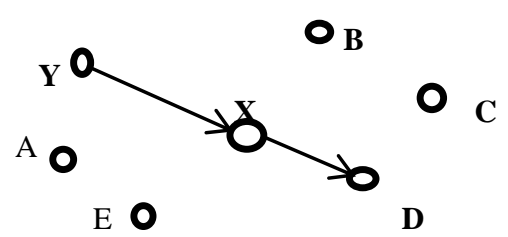

Fig 4 Implementation of Greedy approach in WSN 4.5 Qos-based protocols

In addition to diminishenergy utilization, it is also important to consider quality of service(Qos) condition in terms of delay, reliability, and fault tolerance in routing in WSNs [19].

4.5.1Sequential Assignment Routing (SAR)

SAR is single of the routing protocols for WSNs that introduces the idea of Qos in the routing decisions. This protocol is used for to attain energy efficiency and fault tolerance. Simulation results showed that SAR offers less power consumption [21]. 4.5.2SPEED

SPEED is another QoS routing protocol for sensor networks .The protocol requires each node to preserve information about its neighbors and uses geographic forwarding to finds paths. SPEED protocols avoid congestion when the networks are congested [19].

\subsubsection{MMSPEED}

The abbreviation of MMSPEED is multi-path multi-speed protocol. This protocol is an modified version of SPEED. MMSPEED providing multi path multi speed of packets across the networks. This protocol spread over networks layer and medium access control layer and provides QoS supports in terms of reliability and timeliness [19].

\subsubsection{Energy-Aware QoS Routing Protocol}

This protocol is generated by imaging sensors, imaging sensors are used to produce actual time traffic. Energy aware routing finds a slightest cost and Energy Efficient pathway that meets End to End interruption during its link. The cost of a linkage is function of node's reserved energy, transmission energy, error rate and several other communication parameters. In this protocol a class based queuing model is used for the maintain of real time and best effort traffic while shares the military for real time and non-real time traffic. A list of least cost path is resolute by this protocol by using a wide version of Dijkastra's algorithm and selects a path from that list which satisfies the end to end delay condition [21].

Summarize recent results on data routing in WSN in Table 2. The table shows how different routing protocols fit under different categories and also comparison different routing technique according to many metrics. 


\section{CONCLUSION AND FUTURE SCOPE}

In Recent year, the routing protocol in WSN has become one of the most important research areas and introduces single challenges compared to traditional data routing in wired networks. This paper presents a complete survey of routing techniques in WSN that have been presented in the literature. Overall, the routing techniques are classified structure into different categories: Flat, hierarchical, location based and Qos based routing protocols. Furthermore, these protocols are categorized into multipath-based, Query based, QoS based routing technique depending on protocol operation. Although many of these routing techniques look capable, there are at rest many challenges that need to be solved in sensor networks.

Table 2. Classification and Comparison of Routing Protocols in Wireless Sensors Networks.

\begin{tabular}{|c|c|c|c|c|c|c|c|c|}
\hline $\begin{array}{l}\text { Routing } \\
\text { protocols }\end{array}$ & classification & $\begin{array}{l}\text { Data } \\
\text { Aggregation }\end{array}$ & $\begin{array}{l}\text { Power } \\
\text { Usage }\end{array}$ & Scalability & Multipath & $\begin{array}{l}\text { Query } \\
\text { Based }\end{array}$ & QoS & Mobility \\
\hline LEACH & Hierarchical & yes & Max. & Good & No & No & No & Fixed BS \\
\hline PEGASIS & Hierarchical & No & Max & Good & No & No & No & Fixed BS \\
\hline TEEN & Hierarchical & Yes & Max & Good & No & No & No & Fixed BS \\
\hline APTEEN & Hierarchical & Yes & Max & Good & No & No & No & Fixed BS \\
\hline TTDD & Hierarchical & No & Ltd. & Low & possible & possible & No & Yes \\
\hline GEAR & Location & NO & Ltd. & Ltd. & No & No & No & Ltd. \\
\hline GAF & Location & NO & Ltd. & Good. & No & No & No & Ltd. \\
\hline SPAN & Location & No & N/A & Ltd. & No & No & No & Ltd. \\
\hline $\begin{array}{l}\text { MECN \& } \\
\text { SMECN }\end{array}$ & Location & No & Max. & Low & No & No & No & No \\
\hline SPIN & Flat & Yes & Ltd. & Ltd. & Yes & Yes & No & Possible \\
\hline $\begin{array}{l}\text { Rumor } \\
\text { Routing }\end{array}$ & Flat & Yes & N/A & Good & No & Yes & No & $\begin{array}{l}\text { Very } \\
\text { Ltd. }\end{array}$ \\
\hline ACQUIRE & Flat & Yes & N/A & Ltd. & No & Yes & No & Ltd. \\
\hline COUGAR & Flat & Yes & Ltd & Ltd. & No & Yes & No & No \\
\hline DD & Flat & yes & Ltd. & Ltd. & yes & yes & No & Ltd. \\
\hline SPEED & QoS & No & N/A & Ltd. & No & Yes & Yes & No \\
\hline SAR & QoS & Yes & N/A & Ltd. & No & Yes & Yes & No. \\
\hline
\end{tabular}

\section{ACKNOWLEDGEMENT}

I hereby acknowledge that the above referred work is my own research work and I am entirely responsible for any kind of misuse of material. I thank my mentor Er. Harsukh Singh to guide and encourage me and for putting all her valuable time for me to get the desired output.

\section{REFERENCES}

[1] S. Pant, N. Chauhan, P. Kumar, "Effective Cache based Policies in Wireless Sensor Networks: A Survey," International Journal of Computer Application, vol. 11, No. 10, pp. 17-21, 2010

[2] I. Akyildiz, W. Su, Y. Sankarasubramaniam, E. Cayirci, "Wireless sensor networks: a survey Computer Networks, Elsevier,vol. 38, No. 4, pp. 393-422, 2002.

[3] Zhang, D., Li, G., Zheng, K., \& Ming, X. "AnEnergy Balanced Routing Method Based onForward-Aware Factor for Wireless Sensor Networks",IEEE, vol. 10, No .1,pp. 766773,2014.

[4] L. Jian-qi, C. Bin-fang, W. Li, and W. Wen-Hu, "Energy optimized approach based on clustering routing protocol for wireless sensor networks," Proceeding of $25^{\text {th }}$
Internal Conference on Chinese Control and Decision conference., pp. 3710-3715, 2013.

[5] K. Akkaya, M. Younis, "A survey on routing protocols forwireless sensor networks," Ad Hoc Networks,Elsevier, vol. 3, pp. 325-349, 2005.

[6] S. Choudhary, "A Survey of LEACH Protocol and its Modified Versions in Wireless Sensor Network," International journal of Advanced Research in Computer Science and Software Engineering, vol. 4, No. 1, pp. 850-853, 2014.

[7] P. Nayak , D. Ph, "Comparison of Routing Protocols in WSN using NetSim Simulator: LEACH Vs LEACHC,'International journal of computer Application, vol. 106, No. 11, pp. 1-6, 2014.

[8] H. Kour , A. K. Sharma, "Hybrid Energy Efficient Distributed Protocol for Heterogeneous Wireless Sensor Network," Interntional Journal of Computer Application., vol. 4, No. 6, pp. 1-5, 2010.

[9] K.Singh, "A Survey of Energy-Efficient Hierarchical Cluster-Based Routing in Wireless Sensor Networks," International Journal of Advanced Networking and Applications, vol.2, No. 2, pp. 570-580, 2010 
[10] I. Stojmenovic , X. Lin. "GEDIR: Loop-Free Location Based Routing in Wireless Networks", International Conference on Parallel and Distributed Computing and Systems, Boston, MA, USA, Nov. 3- 6, 1999.

[11] Shagun paul kaur "International Journal of Advanced Research in Computer Science and Software Engineering Review on Existing Load Balancing Techniques of Cloud Computing," vol. 4, No. 2, pp. 168-171, 2014.

[12] S. Yadav and S. S. Yadav, "Review for Leach Protocol in WSN," International Journal of RecentDevelopmentinEngineering andTechnologyVol. 2, No. 6, pp. 69-71, 2014.

[13] A. Manjeshwar ,D. P. Agrawal, "APTEEN: a hybrid protocol for efficient routing and comprehensive information retrieval in wireless sensor networks," Proceding of 16th International Parallel Distribution Processor Symposium, 2002.

[14] G. Dhand, S. S. Tyagi, "Survey on Data-Centric protocols of WSN," International Journal of Application or Innovation in Engineering \& Management, vol. 2, No. 2, pp. 279-284, 2013.

[15] V. S. Patel, C. R. Parekh, "survey on sensor protocol for information via negotiation ( spin ) protocol," International Journal of Research in Engineering and Technology ,pp. 208-211, 2014.

[16] B. Baranidharan,B. Shanthi, "A Survey on Energy Efficient Protocols for Wireless Sensor Networks," International Journal of Computer Application , vol. 11, No. 10 , pp. 35-40, 2010.

[17] M. P. Tyagi, "Comparative Study of Routing Protocols in Wireless Sensor Network," international journal of advanced research in computer science and software engineering., vol. 2, No. 9, pp. 134-140, 2012.

[18] H. Luo, F. A. N. Ye, J. Cheng, S. Lu, and L. Zhang, "TTDD: Two-Tier Data Dissemination in Large-Scale Wireless Sensor Networks,",Wireless Networks, Springer, pp. 161-175, 2005.
[19] R. Sumathi ,M. G. Srinivas, "A Survey of QoS Based Routing Protocols for Wireless Sensor Networks," Journal of Information Process System, vol. 8, No. 4, pp. 589-602, 2012

[20] G. Villalba, S. Orozco, T. Cabrera, B. Abbas, "Routing protocols in wireless sensor networks.," Sensors (Basel)., vol. 9, No. 2, pp. 8399-421, 2009

[21] S.singh,M.singh,D.singh, "Routing protocols in wireless sensor networks.," International Journal of Computer science and Engineering, vol. 1, No. 2, pp. 63- 83, 2010.

[22] S. Tanwar, N. Kumar,J. J. P. C. Rodrigues, "A systematic review on heterogeneous routing protocols for wireless sensor network," Journal of Networking Computer Appication., vol. 53, pp. 39-56, 2015.

[23] M.Yassein, A. Al-zou, Y. Khamayseh,W. Mardini, "Improvement on LEACH Protocol of Wireless Sensor Network ( VLEACH )," International Journal of Digital Content Technology and its Applications,Vol.3.No.2, pp. 132-136,2009.

[24] B.Manzoor, N. Javaid, O. Rehman, M. Akbar, Q. Nadeem, A. Iqbal, and M. Ishfaq, "Q-LEACH : A New Routing Protocol for WSNs,",Proceding of Computer Science, ,Elsevier, vol. 19, pp. 926-931, 2013

[25] V.Geetha, P. V Kallapur, S. Tellajeera, "Clustering in Wireless Sensor Networks : Performance Comparison of LEACH \& LEACH-C Protocols Using NS2,"procedia technology,Elsevier, vol. 4, pp. 163-170, 2012.

[26] M. Ghiasabadi, M. Sharifi, N. Osati, S. Beheshti, and M. Sharifnejad, "TEEN: a routing protocol for enhanced efficiency in wireless sensor networks," proceeding of IEEE, vol. 1, No. C, pp. 2009-2015, 2001.

[27] A. Ahlawat , V. Malik, "An extended vice-cluster selection approach to improve $\mathrm{v}$ leach protocol in WSN,"proceeding of $3^{\text {rd }}$ International Conference of Advanced. Computing and Commun. Technology,IEEE, pp. 236-240, 2013. 